\title{
Mapping the Media Landscape \\ The influence of ownership and broadcast schemes on modern news consumption
}

\author{
Jérémie Rappaz \\ EPFL \\ Switzerland \\ jeremie.rappaz@epfl.ch
}

\begin{abstract}
Information about world events is disseminated through a wide variety of news channels, each with specific considerations in the choice of their reporting. Although the multiplicity of these outlets should ensure a variety of viewpoints, recent reports suggest that the rising concentration of media ownership may void this assumption. This observation motivates the study of the impact of ownership on the global media landscape and its influence on the coverage the actual viewer receives.

In this talk, I will present a comprehensive methodology to analyze the news landscape on a global scale. In particular, I will present our most recent line of work that maps the editorial line evolution of thousands of news channels over the past years. I will showcase several examples of important changes in programming induced by mergers and acquisitions revealed by this approach. I will also discuss the implications of our work by describing the milestone of our newly established initiative.
\end{abstract}

\section{Introduction}

Information is no longer a scarce resource. The digitization of information pathways has rendered the news more freely accessible at scale, even shifting consumption to an active process, where readers share, react or create the content directly. This transformation has induced several changes:

The devaluation of news as information is initiated by the imperative to be aware of world's affairs through the description of events rather than their analysis. The endless stream of notifications and factoids is nearly instantaneously and endlessly broadcast, overwhelming the reader with information without context.

The experiential shift in news consumption began with the ability to share, comment and vote on news items. It has empowered the public to participate directly in the news cycle, potentially shaping narratives and biasing the coverage of certain events by artificially reinforcing their importance. This reduces the barrier of entry for rumors,

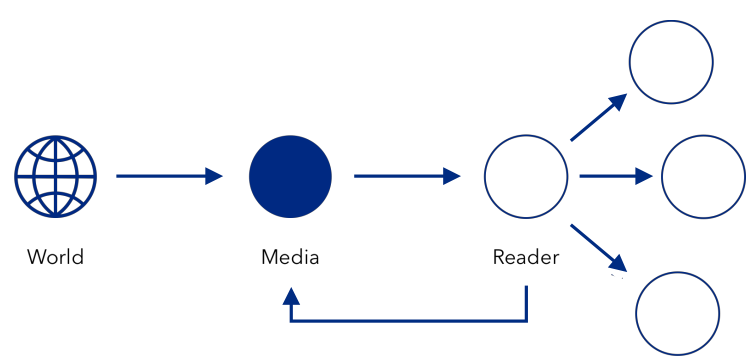

Figure 1: The digitization of information distribution and consumption has modified the news feedback loop. From the World to the Media, a selection process occurs. This subset of reported events is then broadcast to the Reader, who redistributes the information but also impacts the production of future event selections through a feedback loop with the Media.

misleading, doctored, fake or otherwise deceptive content. The economic incentives of the attention economy have also driven down the cost of their creation and upped their economic value, further evidence that market forces can directly influence content creation patterns.

The increased readership uncertainty about the quality of reported news and the inability for consumers to properly assess it can depress the market, as classic economic theories suggest. It is also worth noting that this burden has been transferred from news organizations or regulators down to the reader itself. Hence, the ability to evaluate the quality and origin of coverage is essential to the integrity of news as an information source.

This apparent lack of transparency directly motivates the development of novel methods, designed to harness the very nature of our new information broadcasting processes. This talk presents recent research trends aimed at monitoring the news landscape and describe the steps necessary to make these available to the public.

The talk is structured in three parts. (1) I will discuss the importance of having an open and transparent access to historical media coverage data in order to foster media accountability. (2) I will describe a methodology to estimate similarities across news channels and will showcase several real-world example of what is observable 


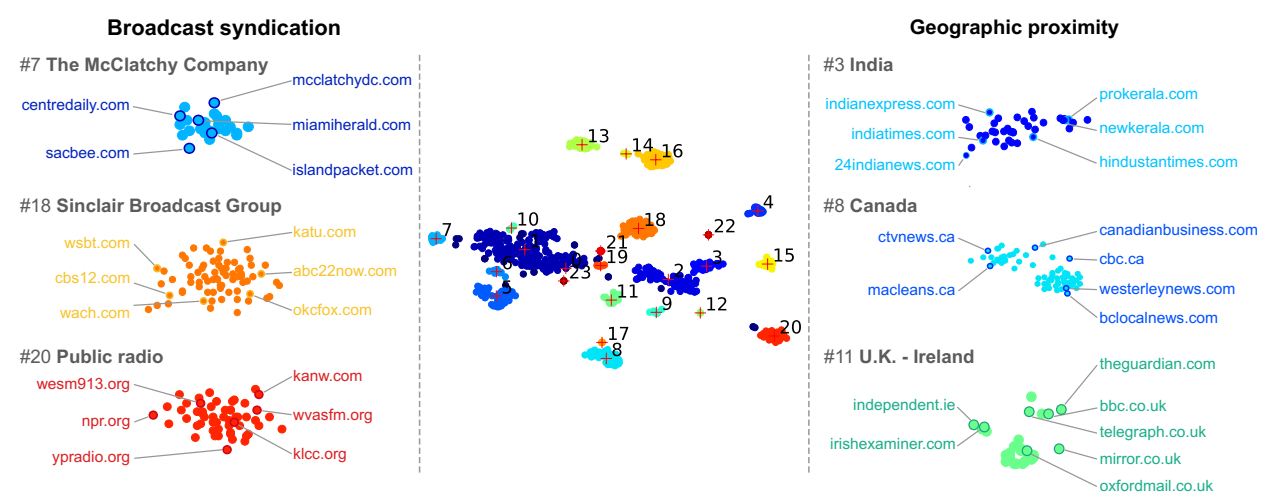

Figure 2: A map of source similarity computed based on event coverage patterns (Bourgeois et al., 2018).

through such methods. (3) I will share the realworld implications of our work and present our recently established Media Observatory Initiative, ${ }^{1}$ concluding with future research directions.

The rest of this paper summarizes recent research results that will be detailed during the talk.

\section{Mapping the News Landscape}

In (Bourgeois et al., 2018), we leverage the fact that news entities have to select and filter the coverage they broadcast through their respective channels. The subjective nature of this filtering induces biases due to, among other things, resource constraints, editorial guidelines, ideological affinities, or even the fragmented nature of the information at a journalist's disposal. In this work, we introduced a methodology to capture the latent structure of medias decision process at a large scale, producing a map of source's similarities based on their selection of reported events, as shown in Fig. 2, estimating biases for which there is no ground truth.

Temporal Dynamics In (Rappaz et al., 2019), we pursue this line of thought by measuring the evolution of the media landscape as a dynamic system. The diversity of this ecosystem is a key requirement for the readership to be exposed to a wide variety of opinions and ideas. It should stem from the natural competition between media content suppliers. However, we identify acquisition campaigns conducted by large news organizations which cause visible content alignment. This strong consolidation of resources, on top obfuscating the origin of a news, can bias the perception of an event's significance.

\section{Leveraging the Map}

Detecting Influence Uncovering patterns in coverage allows the method to detect similarities between sources. While many are benign, from common geographical considerations to some forms on content consolidation, others raise questions as to the authenticity of reporting. Indeed, we show that corporate affiliations can be strong drivers of content similarity, even when the reader is exposed to different downstream broadcasters. Indeed, many large groups franchise household names, such as $\mathrm{ABC}$ or Fox, while retaining editorial control, unbeknownst to the news consumer. One famous instance was recent put into the spotlight with Sinclair Broadcast Group.

Increasing Diversity While the map illustrates similarity between news sources, the metric can also be reversed to increase the diversity of coverage in a particular selection of reporting. For example, an article recommendation system could also be forced to sample from sources that don't usually cover the same sets of events as the one currently being shown. We propose a method that can select a diverse set of sources while also retaining important events. This is done in a parametric way, so the ratio of relevance to novelty can be controlled, potentially by the user itself.

\section{References}

Dylan Bourgeois, Jérémie Rappaz, and Karl Aberer. 2018. Selection bias in news coverage: learning it, fighting it. In Companion of the The Web Conference 2018 on The Web Conference 2018.

Jérémie Rappaz, Dylan Bourgeois, and Karl Aberer. 2019. A dynamic embedding model of the media landscape. In The World Wide Web Conference.

\footnotetext{
${ }^{1}$ https: //mediaobservatory.com
} 